\section{FLUID MOTION}

\section{Theoretical Hydrodynamics}

By L. M. Milne-Thomson. Fifth edition, revised and enlarged. Pp. xxii $+743+4$ plates. (London: Macmillan and Co., Ltd., 1968.) 1058.

THe fifth edition of Professor Milne-Thomson's internationally known book contains interesting new material not included in the fourth edition of 1960 . The new features include a chapter bringing together various exact treatments of two-dimensional motion with a free surface in a gravitational field, followed by one dealing with approximations (mostly linearized) relevant to this work, but with emphasis on waves; a chapter on tensor methods applied to the flow of viscous fluids; a chapter on flow with small Reynolds' number, including an account of a novel application of the complex variable to Stokes' flow; and an outline of the theory of two-dimensional laminar flow in a boundary layer.

This well known book indicates the mathematician's approsch to investigations of fluid motion. It does not concern itself with the experimental or practical side of the associated phenomena, but deals primarily with the underlying mathematical theory and its development. One must, however, beware of falling into the trap of thinking that the overall theory of fluid motion is as tidy and as elegant as Professor Milne-Thomson's mathematical exposition may lead the uninitiated to expect. Much remains to be investigated. Professor Milne-Thomson does, however, make contact with experimental work in many places and indicates, briefly, the principal concepts which have evolved from experimental work. These serve as valuable introductions to the mathematical development.

The stated purpose of the book is to give a thorough, clear and methodical introductory exposition to the mathematical theory of fluid motion which will be useful in applications to both hydrodynamics and aerodynamics, and the author's objective is certainly achieved. It should not be overlooked, however, that there are many mathematically formulated problems in theoretical hydrodynamics which defy mathematical solution, but which, nevertheless, can be made to yield valuable approximate numerical answers when treated by the very powerful methods of numerical analysis and electronic computation.

Every engineer, physicist or mathematician who teaches or works in the field of theoretical hydrodynamics should have a copy of this compendium in his private library, and copies of the book ought to be made easily available to all undergraduate or postgraduate students who are studying or carrying out original investigations in this field.

L. Rosenhead the authority of the Church. This intention is not apparent in the original edition of the book, but is quite explicit in the much expanded version of it inserted in a volume of the collected edition of his works which appeared in Geneva in 1771.

The volume under review is a facsimile reprint of all English translation of Voltaire's treatise, published in London in the same year as the original. It is part of a series called "Library of Science Classics"; frankly, this is stretching the meaning of such an expression to the point of extravagance, and to have selected for reprint an obscure English version rather than the French text only makes matters worse-for the scientific value of Voltaire's dilettantish effort is nil, and its only merit derives from the incisiveness and elegance of his inimitable style, the purest form ever attained by the French language. In fact, even the pedantic "Teacher of the Mathematicks" who made the translation could not entirely suppress the spell of Voltaire's style; it still glimmers through the dullness of his version, enough perhaps to give an idea of its brilliance to an American reader.

From the point of view of the social relations of science, however, this English translation is a revealing document. Voltaire addressed himself to a refined intelligentsia, whose energy was directed towards the reform of the political institutions; the patrons of his English translator had their political struggle behind them and were busily engaged, with remarkable clearsightedness, in exploiting the resources of science for promoting an expanding industrial production. The most prominent among them had not to wait for Voltaire, of course, in order to acquaint themselves with Newton's work. Some would read the Principia in Motte's translation, others would be content with Pemberton's simpler exposition. Our teacher of mathematics, however, coped with the lesser fry; this is illustrated by the catalogue of "hard words" appended to his translation: "Convex is the Outside of a curve Line, or Superfice. Concave, the Inside thereof. Cone is a solid Figure, wherein its circular Base tapers, till it come to a Point, as a Sugar Loaf"; or, somewhat unexpectedly. "Plenum is a Space quite void of Matter". That people in need of such explanations would at all want to gain some familiarity with Newtonian philosophy is a telling testimony of the intellectual climate of the eighteenth century British craftsmen. It is curious to reflect, in this connexion, that at the same time the Cambridge undergraduates learned physics from Rohault's Cartesian textbook, but in an edition spiced with footnotes written by Clarke, through which the Newtonian concepts were smuggled into the curriculum.

L. Rosenfeld

\section{VOLTAIRE ON NEWTON}

The Elements of Sir Isaac Newton's Philosophy By Voltaire. Translated by John Hanna. (Cass Library of Science Classies, No. 2.) Pp. xvi+368. (London: Frank Cass and Co., Ltd., 1967. Originally published in 1738.) $90 s$.

IN his campaign against the absolutism of the French government, one of Voltaire's favourite moves was to contrast French conditions with those prevailing across the Channel, which he painted in rosier colours than a sober report would allow. Thus, he was led to oppose debased Cartesianism predominant in France to the vigorous experimental philosophy created by Newton's genius, and to this end he wrote in 1738 a popular exposition of Newton's optical and astronomical discoveries. By acquainting the French public with the main facts on which the new philosophy was founded. Voltaire intended to make them more receptive for the deism he was propagating as a spiritual weapon against

\section{LASERS IN MEDICINE}

Biomedical Aspects of the Laser

The Introduction of Laser Applications into Biology and Medicine. By Leon Goldman. Pp. viii + 232. (Berlin and New York: Springer-Verlag, 1967.) 45.60 DM. \$11.40.

THE laser ("Light Amplification by Stimulated Emission of Radiation") is already familiar to the general public as an exciting new tool, the possible uses of which are as diverse as its power is great; but because of the latter and because its dangers are as yet so unassessed, its actual deployment on all but a limited scale still awaits us in almost every field.

The concept of this stimulated emission dates from the work of Einstein at the close of the First World War. but only in the past decade have we discovered adequate means of producing such emission in the micro-wave area near optical regions of the spectrum. The atoms of the laser medium (chromium in the most widely used ruby laser, neodymium in glass, argon, carbon dioxide and so on) must absorb a sufficient amount of energy at the 